\title{
A deficiency in cold-inducible RNA-binding protein accelerates the inflammation phase and improves wound healing
}

\author{
JUAN PABLO IDROVO $^{1 *}$, ASHA JACOB ${ }^{1,2^{*}}$, WENG LANG YANG ${ }^{1,2}$, ZHIMIN WANG $^{1,2}$, HAO TING YEN ${ }^{1,2}$, \\ JEFFREY NICASTRO $^{1}$, GENE F. COPPA ${ }^{1}$ and PING WANG ${ }^{1,2}$
}

${ }^{1}$ Department of Surgery, Hofstra North Shore-LIJ School of Medicine and ${ }^{2}$ Center for Translational Research, The Feinstein Institute for Medical Research, Manhasset, NY, USA

Received September 22, 2015; Accepted December 23, 2015

DOI: $10.3892 /$ ijmm.2016.2451

\begin{abstract}
Chronic or non-healing wounds are a major concern in clinical practice and these wounds are mostly associated with diabetes, and venous and pressure ulcers. Wound healing is a complex process involving overlapping phases and the primary phase in this complex cascade is the inflammatory state. While inflammation is necessary for wound healing, a prolonged inflammatory phase leads to impaired healing. Cold-inducible RNA-binding protein (CIRP) belongs to a family of cold-shock proteins that are expressed in high levels under stress conditions. Recently, we demonstrated that a deficiency in CIRP led to decreased inflammation and mortality in an experimental model of hemorrhagic shock. Thus, we hypothesized that a deficiency in CIRP would accelerate the inflammatory phase and lead to an improvement in cutaneous wound healing. In this study, to examine this hypothesis, a full-thickness wound was created on the dorsum of wild-type (WT) and $\mathrm{CIRP}^{-/-}$mice. The wound size was measured every other day for 14 days. The wound area was significantly decreased in the $\mathrm{CIRP}^{-/}$mice by day 9 and continued to decrease until day 14 compared to the WT mice. In a separate cohort, mice were sacrificed on days 3 and 7 after wounding and the skin tissues were harvested for histological analysis and RNA measurements. On day 3 , the mRNA expression of tumor necrossis factor (TNF)- $\alpha$ in the skin tissues was increased by 16 -fold in the WT mice, whereas these levels were increased by 65 -fold in the $\mathrm{CIRP}^{-/}$mice. Of note on day 7, while the levels of TNF- $\alpha$ remained high in the WT mice, these levels were significantly decreased in the $\mathrm{CIRP}^{-/-}$mice. The histological analysis of the wounded skin tissue indicated an improvement as early as day 3 in the $\mathrm{CIRP}^{-/}$mice, whereas
\end{abstract}

Correspondence to: Professor Ping Wang, Department of Surgery, Hofstra North Shore-LIJ School of Medicine, 350 Community Drive, Manhasset, NY 11030, USA

E-mail: pwang@nshs.edu

"Contributed equally

Key words: cutaneous wounds, cold-inducible RNA-binding protein, cold-inducible RNA-binding protein knockout, inflammation, angiogenesis in the WT mice, infiltrated immune cells were still present on day 7. On day 7 in the CIRP $^{-/}$mice, Gr-1 expression was low and CD31 expression was high, whereas in the WT mice, Gr-1 expression was high and CD31 expression was low, indicating that the $\mathrm{CIRP}^{-/}$mice have already moved into the angiogenesis and tissue formation phase, whereas the WT mice were still in the inflammatory state. These data collectively suggest that a deficiency in CIRP accelerates the wound healing process.

\section{Introduction}

Chronic lower extremity wounds are an important health concern and the prevalence of these is close to $1.3 \%$ in the population $(1,2)$. These wounds are most likely associated with venous disease, diabetes and pressure. The most common chronic wound is the venous leg ulcer which accounts for $40-70 \%$ of lower extremity wounds (3). A significant number of individuals with venous leg ulcers suffer from impaired mobility, depression, social anxiety and a poor self esteem. Chronic wounds are also significantly associated with diabetes; close to $15 \%$ of the diabetic population develop diabetic foot ulcers, which may result in lower extremity amputations (4). These conditions have a profound effect on the quality of life of patient and mortality rates, as well as health care costs $(2,3,5)$. Understanding the mechanisms of wound healing is pertinent in developing therapeutic strategies for the treatment of chronic wounds.

Wound healing is comprised of an intricate balance of distinct but overlapping phases of inflammation and resolution, leading to tissue repair and tissue remodeling. Immediately after wounding, the initial phase of wound healing, the inflammatory phase begins; neutrophils infiltrate the wound area and initiate the process of debridement of devitalized tissue. To invade microbes they release reactive oxygen species (ROS), antimicrobial peptides, proteases and form extracellular traps. These short-lived neutrophils are then engulfed by resident macrophages and thus the inflammatory stage subsides. The second phase is the tissue formation, where epithelialization and newly formed granulation tissue consisting of endothelial cells, macrophages and fibroblasts tend to cover the wound area and restore tissue integrity. The third phase is the tissue remodeling one in which extracellular matrix molecules form and cell-cell interactions occur, and eventually facilitate wound healing (6-8). If, however, the inflammatory stage is prolonged, 
there is an impairment in wound healing. Chronic non-healing or slow-healing wounds are often caused by exaggerated inflammation due to the persistence of neutrophils in tissue (9).

Cold-inducible RNA-binding protein (CIRP) belongs to a family of cold-shock proteins and is constitutively expressed in low levels in a variety of tissues (10-12). It is, however, highly expressed during hypothermia, hypoxia and ultraviolet radiation (10,13-15). Recently, we demonstrated that CIRP expression is increased in the blood of patients who were admitted to intensive care units due to hemorrhagic shock (16). In animal models, deficiency in CIRP led to decreased inflammation and mortality after hemorrhagic shock (16). Wound healing is compromised by a prolonged inflammatory phase, as observed in chronic wounds, which results in exaggerated inflammation. Thus, we hypothesized that a deficiency in CIRP would improve cutaneous wound healing.

In order to examine this hypothesis, in this study, we aimed to determine whether a deficiency in CIRP would accelerate the inflammation phase and improve wound healing. We created wounds on the dorsum of wild-type (WT) and CIRPknockout $\left(\mathrm{CIRP}^{-/}\right)$mice and examined the wound healing process.

\section{Materials and methods}

Experimental animals. Male adult (8-10 weeks old) C57BL/6 (WT) mice were purchased from Taconic (Albany, $\mathrm{NY}$, USA). In total, $4 \mathrm{C} 57 \mathrm{BL} / 6$ mice were included in the wound closure experiments conducted to generate the data shown in Fig. 1 and 4-6 mice were included for the data generated from the day 3 and day 7 experiments. The mice were allowed to acclimatize for 5 days in a temperature-controlled environment with a 12-h light/dark cycle and fed a standard chow diet and were allowed access to water ad libitum. A male adult $\mathrm{CIRP}^{-/}$mouse (8-10 weeks old) was a gift from Dr Jun Fujita (Kyoto University, Kyoto, Japan) and was bred in our facility for experimental purposes. All experiments were conducted in accordance with the Guide for the Care and Use of Laboratory Animals from the National Institutes of Health. The protocol was approved by the Institutional Animal Care and Use Committee of the Feinstein Institute for Medical Research, Manhasset, NY, USA.

Mouse model of dorsal cutaneous wounds. On the day of the surgery, the mice were anesthetized by isoflurane inhalation, dorsum-shaved and washed with $10 \%$ povidone-iodine. A $2.0 \mathrm{~cm}$ full-thickness circular wound was created on the dorsum using a trephine. The wounds were extended to the muscle layer, but the panniculus carnosas was kept intact. Prior to the skin incision, $1 \%$ lidocaine was injected intradermally at the surgical site. Bleeding was stopped by compression and the wounds were covered with a dressing (Tegaderm) and changed every other day until the end of experiment.

Measurement of wound size. The wound area was traced on a transparency film every other day until day 14 and wound closure was calculated using NIH ImageJ software. The wound closure rate was calculated according to the following formula as described previously (17): wound closure rate $(\%)=\left[\left(\right.\right.$ Area $_{\text {day } 0}-$ Area $\left._{\text {day } n}\right) /$ Area $_{\text {day } 0}$ ] x100; where Area $_{\text {day } 0}$ is the initial wound area on day 0 and the Area $_{\text {day } n}$ is the area on day ' $n$ ' after wounding.
Reverse transcription-quantitative polymerase chain reaction (RT-qPCR). On days 3 and 7 after wounding, the mice were euthanized by $\mathrm{CO}_{2}$ asphyxiation. Skin tissues $1-2 \mathrm{~mm}$ in length and in thickness beyond the wound edge and away from the center were excised and harvested. One half was frozen in liquid nitrogen and the other half was immersed in $10 \%$ formalin. Total RNA was extracted from the frozen tissues and formalin-fixed tissues was used for histological assessment. Total RNA was extracted from the skin tissues using TRIzol reagent (Invitrogen, Carlsbad, CA, USA) and reverse transcribed into cDNA and subjected to RT-qPCR using the $\triangle \Delta \mathrm{Cq}$ method with primers specific for mouse TNF- $\alpha$. The PCR reaction was carried out as described previously (17). The levels were normalized against those of mouse $\beta$-actin. The primer sequences used were as follows: mouse TNF- $\alpha$ forward, 5'-AGA CCC TCA CAC TCA GAT CAT CTT C-3' and reverse, 5'-TTG CTA CGA CGT GGG CTA CA-3' and mouse $\beta$-actin forward, 5'-CGT GAA AAG ATG ACC CAG ATC A-3' and reverse, 5'-TGG TAC GAC CAG AGG CAT ACA G-3'.

Histological and immunohistochemical analyses. The skin tissues were fixed in $10 \%$ buffered formalin, paraffinembedded and sectioned into $5-\mu \mathrm{m}$-thick sections. The sections were stained with hematoxylin and eosin (H\&E) and examined under a light microscope (Nikon Eclipse Ti-S; Nikon Instruments, Inc., Melville, NY, USA). The paraffin-embedded skin tissue was deparaffinized, immunostained with either anti-Gr-1 antibody (Cat no. 108402; Biolegend, San Diego, CA, USA) or anti-CD31 antibody (Cat no. sc-1506; Santa Cruz Biotechnology, Inc., Dallas, TX, USA) and detected with NovaRED substrate (Vector Laboratories, Burlingame, CA, USA) as described previously (18).

Statistical analysis. All data are expressed as the mean values \pm SEM ( $n=4-6$ animals/group) and analyzed by one-way analysis of variance (ANOVA) and compared using the StudentNewman-Keuls (SNK) test for multiple comparisons and the Student's t-test for comparisons between 2 groups. The differences in values were considered significant with a value of $\mathrm{P}<0.05$.

\section{Results}

Wound closure rate is accelerated in $C I R P^{-/-}$mice. We first determined the rate of wound closure in the $\mathrm{CIRP}^{-/}$as compared to the WT mice. As early as day 3, there were visual differences in the wounds between the 2 groups of mice and the wound area also began to decrease in size in the $\mathrm{CIRP}^{-/-}$mice (Fig. 1). This difference in the size of the wound area between the 2 groups of mice was more evident by day 9 ; the size of the wound area was significantly decreased in the $\mathrm{CIRP}^{-/-}$mice $(\mathrm{P}=0.007)$. The size of the wound area remained significantly smaller in the $\mathrm{CIRP}^{-/-}$mice from days 9 to 14 and the greatest significant difference between the 2 groups of mice was observed on day $14(\mathrm{P}<0.001)$. These results demonstrated that a deficiency in CIRP accelerated the cutaneous wound closure rate.

Wound-associated inflammation is resolved early in CIRP $P^{-/}$ mice. To determine the resolution of inflammation after wounding, the mRNA expression of TNF- $\alpha$ in the wounded skin tissue was assessed. In the WT mice, TNF- $\alpha$ expression 
A

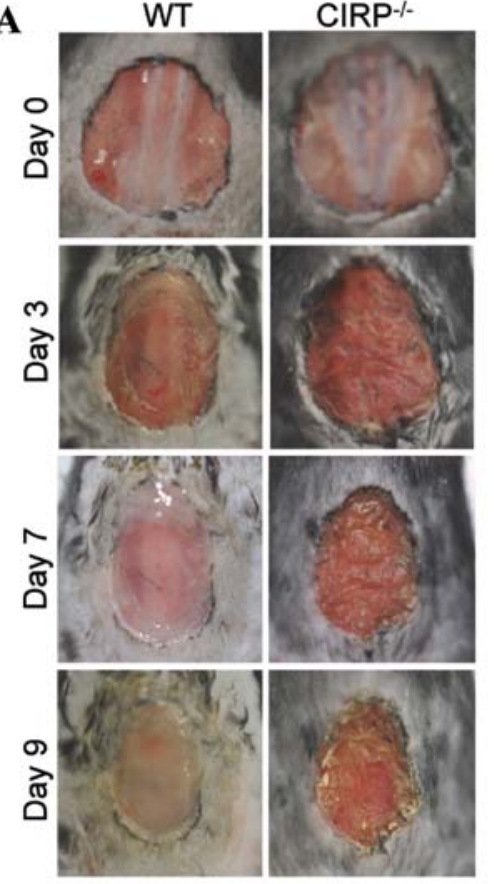

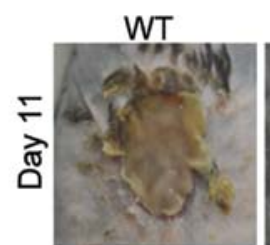

CIRP -1
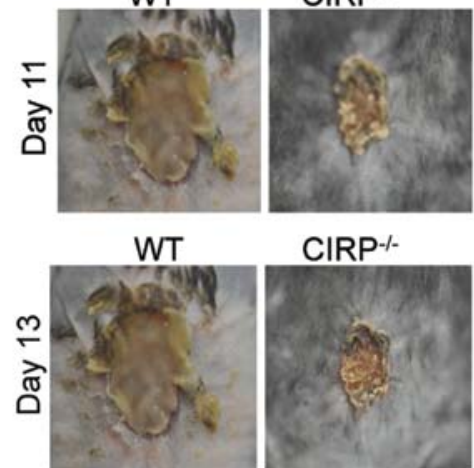

CIRP $\%$

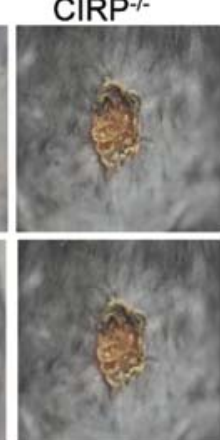

B

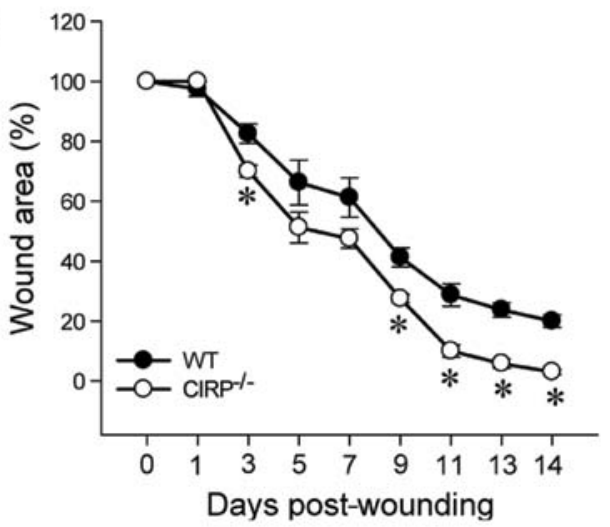

Figure 1. The wound closure rate is accelerated in cold-inducible RNA-binding protein (CIRP) ${ }^{-/-}$mice. Cutaneous wounds created on wild-type (WT) and $\mathrm{CIRP}^{-/}$mice were measured to determine the size of the wound area every other day until day 14. (A) Photographical representation of a single mouse from the 2 groups on different days. (B) Wound closure rate assessed by measuring the wound size as described in Materials and methods and calculated using NIH ImageJ software. Data represented are the means \pm SEM ( $n=4$ /group) and compared by the Student's t-test. " P $<0.05$ vs. WT mice.

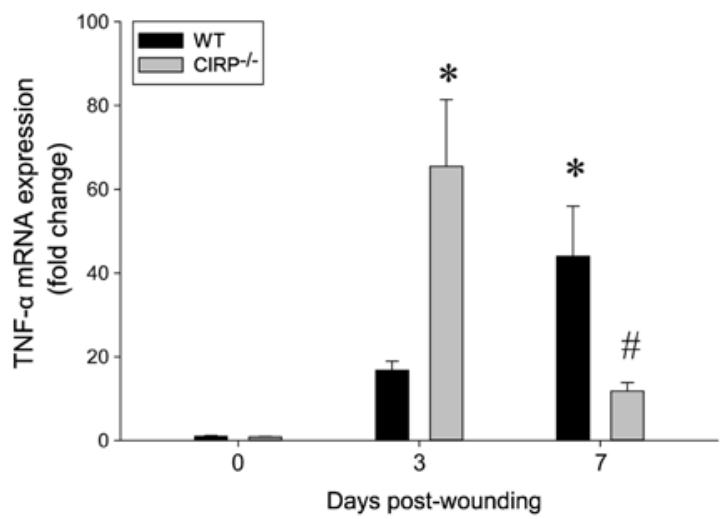

Figure 2. The resolution of inflammation was observed early in cold-inducible RNA-binding protein (CIRP) ${ }^{-/}$mice. Total RNA from skin tissues from the wound on different days from wild-type (WT) and $\mathrm{CIRP}^{-/-}$mice were examined for TNF- $\alpha$ mRNA expression by RT-qPCR. Data represent the means \pm SEM $(n=3-5 /$ group) and were compared by one-way ANOVA and Student-Newman-Keuls test. ${ }^{*} \mathrm{P}<0.05$ vs. WT mice on day $3,{ }^{\text {"P }} \mathrm{P}<0.05$ vs. $\mathrm{CIRP}^{-/}$mice on day 3 . was significantly increased by 16 -fold on day 3 . In the $\mathrm{CIRP}^{-1-}$ mice, while TNF- $\alpha$ expression was also increased by day 3 , the levels were increased by 65 -fold (Fig. 2). Of note, while TNF- $\alpha$ expression was further increased by day 7 in the WT mice, these levels were reduced in the $\mathrm{CIRP}^{-/}$mice (Fig. 2). The decrease in these levels on day 7 presented a statistically significant difference from the levels on day 3 in the WT and $\mathrm{CIRP}^{-/-}$mice.

Histological integrity after wounding is improved in CIRP-/mice. Histological integrity was assessed by H\&E staining of the skin tissue obtained from the wounds on days 3 and 7 in the WT and $\mathrm{CIRP}^{-/-}$mice. In the WT mice on day 3, there was a major disorganization of the dermis layer and the appearance of the infiltration of immune cells into the wounded tissue, whereas in the $\mathrm{CIRP}^{-/-}$mice, the dermis layer was more organized and the tissue appears more intact (Fig. 3). On day 7, granulation tissue began to form and the infiltration of inflammatory cells was observed in both the WT and CIRP ${ }^{-/-}$mice; 

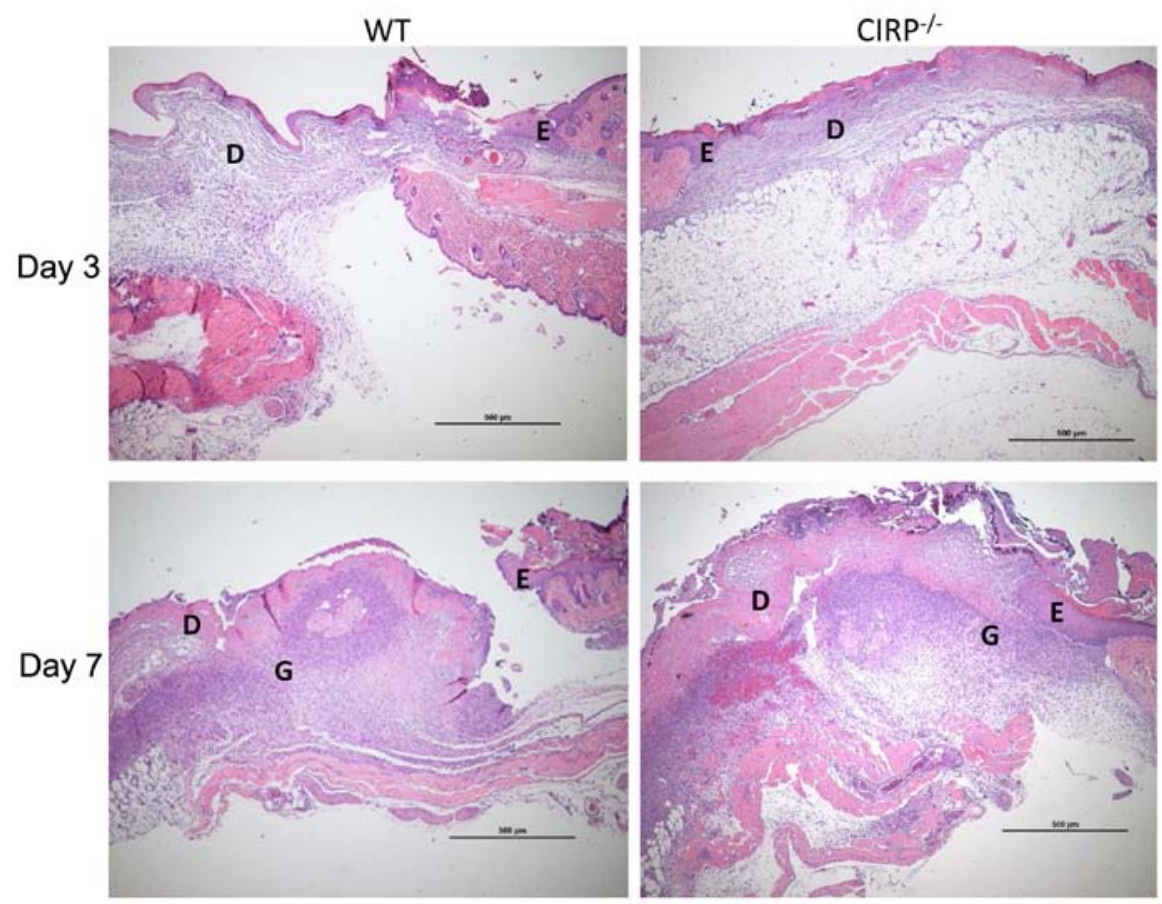

Figure 3. Histological integrity was improved in cold-inducible RNA-binding protein (CIRP) ${ }^{-/}$mice. Skin tissues obtained from the wounds of wild-type (WT) and $\mathrm{CIRP}^{-/-}$mice (day 3 and 7) were sectioned, stained with hematoxylin and eosin, and examined under a light microscope. Representative images at $\mathrm{x} 40$ magnification are shown. E, epidermis layer, D, dermis layer; and G, granulation tissue.
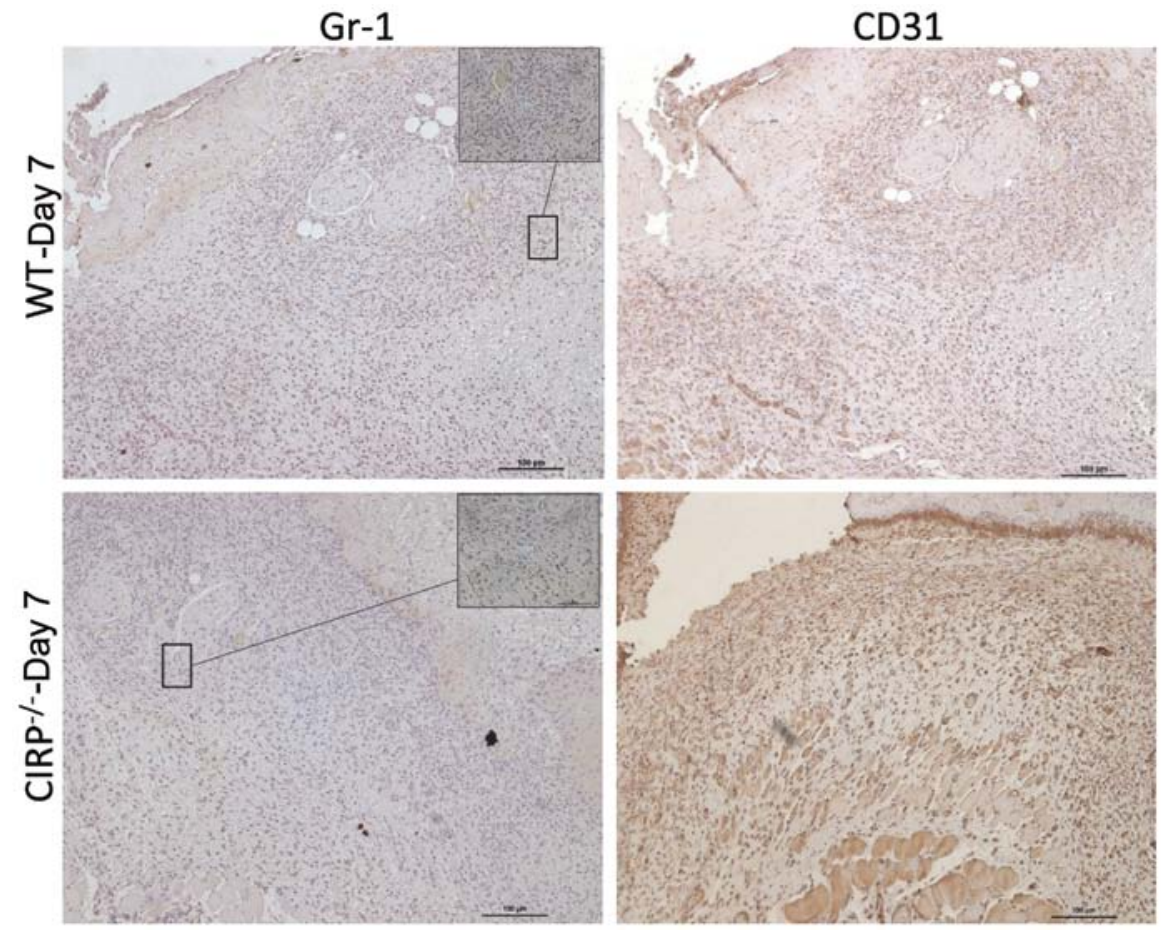

Figure 4. Wound healing was observed early in cold-inducible RNA-binding protein (CIRP) ${ }^{-/}$mice. Skin tissues of wild-type (WT) and CIRP ${ }^{-/}$mice wounds (day 7) were sectioned, immunostained with Gr-1 or CD31 and examined under a light microscope. Representative images at x100 magnification are shown. Images at x400 magnification are shown in the insets for Gr-1 staining.

however, the appearance of infiltrated immune cells was more evident in the WT than in the $\mathrm{CIRP}^{-/}$mice.

Wound-associated neutrophil infiltration is attenuated in $\mathrm{CIRP}^{-/-}$mice. To assess neutrophil infiltration into the wounded skin, the tissue sections were stained with Gr-1 antibody, a surface marker of neutrophils. On day 7, compared to the $\mathrm{CIRP}^{-/}$mice, more Gr-1 staining was observed in the WT mice, indicating the persistence of neutrophil infiltration and subsequent inflammation (Fig. 4). 
Wound-associated angiogenesis is accelerated in CIRP ${ }^{-/-}$mice. After the inflammatory phase, the process of wound healing progresses to the angiogenesis phase where endothelial cells from the bone marrow are migrated to the wounded tissue for neovascularization (8). On day 7 after wounding, the tissues were stained with CD31, a marker of angiogenesis. In the $\mathrm{CIRP}^{-/}$mice, there were dense staining of CD31 as compared to the WT mice, indicating that by day 7 , the $\mathrm{CIRP}^{-/-}$mice had already progressed towards the angiogenesis phase, while the WT mice remained at the inflammatory phase of wound healing (Fig. 4).

\section{Discussion}

Chronic or non-healing wounds are very prevalent in clinical practice and most of these wounds are associated with venous insufficiency, diabetes mellitus, pressure necrosis and vasculitis $(19,20)$. Despite their differences in origin, these wounds fail to progress through normal wound repair and remain in the inflammatory state (21). Wound healing involves a complex mechanism of an intricate balance of distinct phases namely, the inflammatory phase, tissue formation and tissue remodeling. The inflammatory response is the initial process in wound healing. Chronic or non-healing wounds are caused by an exaggerated inflammatory response due to the prolonged presence of neutrophils in the wounded area (8). Thus, identifying factors that may be responsible for such neutrophil persistence will be helpful in developing therapeutic strategies against impaired wound healing.

Our study demonstratred that a deficiency in CIRP significantly decreased the size of the wound area as compared to the WT counterpart, indicating the acceleration of wound healing in mice. The wound closure rate correlated with the inflammatory phase by day 3 in both the $\mathrm{WT}$ and $\mathrm{CIRP}^{-/-}$mice, but there was a significant increase in the TNF- $\alpha$ mRNA expression levels in the $\mathrm{CIRP}^{-/-}$mice compared to the WT mice. This increase in TNF- $\alpha$ expression correlated with the increase in neutrophil infiltration into the wounded tissue, as evidenced by Gr-1 staining. By contrast, on day 7, TNF- $\alpha$ expression was significantly decreased in the $\mathrm{CIRP}^{-/}$mice and there was a concomitant decrease in neutrophil infiltration, whereas in the WT mice, TNF- $\alpha$ expression remained high and a considerable level of Gr-1 staining was also present. This acceleration of the resolution of inflammation on day 7 in the $\mathrm{CIRP}^{-/-}$mice correlated with the second phase, i.e., tissue formation, as observed by the increased expression of CD31, as a measure of neovascularization. Finally, the histological integrity was improved as early as day 3 in the $\mathrm{CIRP}^{-/-}$mice, as observed by an organized and intact dermis layer, whereas in the WT mice, the dermis layer was disordered and the appearance of immune cell infiltration was observed even on day 7. These data suggest that a deficiency in CIRP significantly accelerates the wound healing process in mice.

In physiological wound repair, within a few hours of injury, neutrophils which are entrapped and aggregated in the blood, clot, and transmigrate across the endothelial cell wall of blood capillaries which have been activated by pro-inflammatory cytokines, i.e., TNF- $\alpha(8,22)$. Activated endothelial cells express various adhesion molecules for leukocyte adhesion at the wound site (22). The infiltrating neutrophils are the initial components of the inflammatory phase. They not only invade pathogens, but are also involved in tissue degradation and tissue formation (8). Recruited neutrophils begin the debridement of devitalized tissue and phagocytosis of infectious agents. In this period. they release ROS, cationic peptides and proteases and promote wound healing (23). In general, neutrophil infiltration ceases within a few days and the apoptotic neutrophils are engulfed by macrophages at the wound site $(24,25)$. While neutrophil infiltration is essential for wound healing, the persistence of neutrophils at the wound site leads to a prolonged inflammatory state and impaired wound healing (7).

One main factor in causing chronic inflammation in cutaneous wound healing is ROS. Leukocytes recruited to the wounded site are a rich source of ROS (26). Endothelial cells and fibroblasts present in the wound also contribute to the production of $\operatorname{ROS}(27,28)$. In addition to direct damage to the cell membrane and structural proteins of the extracellular matrix, ROS activate various signaling pathways, leading to the upregulation of pro-inflammatory cytokines, such as TNF- $\alpha$ (29). Therefore, the unbalance of the oxidant/antioxidant microenvironment in chronic wounds is responsible for the prolonged inflammatory state and impaired healing. Diabetic hyperglycemia in diabetes or enhanced hydrostatic pressure as in pressure ulcers can enhance the inflammatory response. Tissue hypoxia, bacterial components, foreign agents and necrotic tissue are all local stimuli which sustain the influx of neutrophils and macrophages to the wound site $(8,19,30)$.

The mechanisms through which a deficiency in CIRP accelerates the inflammatory state and the resolution of inflammation in wound healing have not yet been elucidated. CIRP is known to be expressed in high levels during stress conditions such as cold, ultraviolet exposure and hypoxia (13-15). Recently, we demonstrated that hemorrhage associated with the release of TNF- $\alpha$ was significantly reduced in $\mathrm{CIRP}^{-/}$mice and that the blockade of CIRP using antisera to CIRP in experimental models of hemorrhagic shock and sepsis attenuated inflammatory response and mortality (16). In addition, we demonstrated increased circulation levels of CIRP in patients admitted to the surgical intensive care unit with hemorrhagic shock and have identified CIRP as a mediator of inflammation (16). Shock and sepsis are tissue injury indications that are associated with an exaggerated systemic inflammatory response $(31,32)$. It can thus be speculated that the exaggerated inflammatory response in chronic wounds or non-healing wounds is mediated by CIRP. Future studies are warranted to confirm this hypothesis.

In conclusion, in the present study, we demonstrated that a deficiency in CIRP accelerated the wound closure rate and the inflammatory phase, which led to the resolution of inflammation and neovascularization, and in the improvement of histological integrity during the wound healing process. Therefore, CIRP may be responsible for delayed wound healing in disease conditions, such as diabetes, and venous and pressure ulcers. Therapeutic interventions for blocking CIRP may thus prove to be beneficial for the treatment of cutaneous wounds and a more rapid healing process.

\section{Acknowledgements}

The authors would like to thank Dr Michael Kuncewitch (Hofstra North Shore-LIJ School of Medicine, Manhasset, NY, USA), 
Dr Mahendar Ochani and Dr Xiaoling Qiang (The Feinstein Institute for Medical Research, Manhasset, NY, USA) for their expert technical assistance.

\section{References}

1. Bickers DR, Lim HW, Margolis D, Weinstock MA, Goodman C, Faulkner E, Gould C, Gemmen E and Dall T; American Academy of Dermatology Association; Society for Investigative Dermatology: The burden of skin diseases: 2004 a joint project of the American Academy of Dermatology Association and the Society for Investigative Dermatology. J Am Acad Dermatol 55: 490-500, 2006

2. Phillips TJ: Chronic cutaneous ulcers: Etiology and epidemiology. J Invest Dermatol 102: 38S-41S, 1994.

3. Margolis DJ, Bilker W, Santanna J and Baumgarten M: Venous leg ulcer: Incidence and prevalence in the elderly. J Am Acad Dermatol 46: 381-386, 2002.

4. Pecoraro RE, Reiber GE and Burgess EM: Pathways to diabetic limb amputation. Basis for prevention. Diabetes Care 13: 513-521, 1990.

5. Abbade LP and Lastória S: Venous ulcer: Epidemiology, physiopathology, diagnosis and treatment. Int J Dermatol 44: 449-456, 2005.

6. Diegelmann RF: Excessive neutrophils characterize chronic pressure ulcers. Wound Repair Regen 11: 490-495, 2003.

7. Dovi JV, Szpaderska AM and DiPietro LA: Neutrophil function in the healing wound: Adding insult to injury? Thromb Haemost 92: 275-280, 2004.

8. Eming SA, Krieg $\mathrm{T}$ and Davidson JM: Inflammation in wound repair: Molecular and cellular mechanisms. J Invest Dermatol 127: 514-525, 2007.

9. Jun JI, Kim KH and Lau LF: The matricellular protein CCN1 mediates neutrophil efferocytosis in cutaneous wound healing. Nat Commun 6: 7386, 2015.

10. Nishiyama H, Higashitsuji H, Yokoi H, Itoh K, Danno S, Matsuda T and Fujita J: Cloning and characterization of human CIRP (cold-inducible RNA-binding protein) cDNA and chromosomal assignment of the gene. Gene 204: 115-120, 1997.

11. Nishiyama H, Danno S, Kaneko Y, Itoh K, Yokoi H, Fukumoto M, Okuno H, Millán JL, Matsuda T, Yoshida $\mathrm{O}$ and Fujita $\mathrm{J}$ : Decreased expression of cold-inducible RNA-binding protein (CIRP) in male germ cells at elevated temperature. Am J Pathol 152: 289-296, 1998.

12. Nishiyama H, Xue JH, Sato T, Fukuyama H, Mizuno N, Houtani T, Sugimoto T and Fujita J: Diurnal change of the cold-inducible RNA-binding protein (Cirp) expression in mouse brain. Biochem Biophys Res Commun 245: 534-538, 1998.

13. Sheikh MS, Carrier F, Papathanasiou MA, Hollander MC, Zhan Q, Yu K and Fornace AJ Jr: Identification of several human homologs of hamster DNA damage-inducible transcripts. Cloning and characterization of a novel UV-inducible cDNA that codes for a putative RNA-binding protein. J Biol Chem 272: 26720-26726, 1997.

14. Wellmann S, Bührer C, Moderegger E, Zelmer A, Kirschner R, Koehne P, Fujita J and Seeger K: Oxygen-regulated expression of the RNA-binding proteins RBM3 and CIRP by a HIF-1-independent mechanism. J Cell Sci 117: 1785-1794, 2004.
15. Xue JH, Nonoguchi K, Fukumoto $M$, Sato T, Nishiyama $H$, Higashitsuji $\mathrm{H}$, Itoh $\mathrm{K}$ and Fujita J: Effects of ischemia and $\mathrm{H}_{2} \mathrm{O}_{2}$ on the cold stress protein CIRP expression in rat neuronal cells. Free Radic Biol Med 27: 1238-1244, 1999.

16. Qiang X, Yang WL, Wu R, Zhou M, Jacob A, Dong W, Kuncewitch M, Ji Y, Yang H, Wang H, et al: Cold-inducible RNA-binding protein (CIRP) triggers inflammatory responses in hemorrhagic shock and sepsis. Nat Med 19: 1489-1495, 2013.

17. Idrovo JP, Yang WL, Jacob A, Ajakaiye MA, Cheyuo C, Wang Z, Prince JM, Nicastro J, Coppa GF and Wang P: Combination of adrenomedullin with its binding protein accelerates cutaneous wound healing. PLoS One 10: e0120225, 2015.

18. Giangola MD, Yang WL, Rajayer SR, Nicastro J, Coppa GF and Wang P: Growth arrest-specific protein 6 attenuates neutrophil migration and acute lung injury in sepsis. Shock 40: 485-491, 2013.

19. Singer AJ and Clark RA: Cutaneous wound healing. N Engl J Med 341: 738-746, 1999.

20. Gosain A and DiPietro LA: Aging and wound healing. World J Surg 28: 321-326, 2004

21. Loots MA, Lamme EN, Zeegelaar J, Mekkes JR, Bos JD and Middelkoop E: Differences in cellular infiltrate and extracellular matrix of chronic diabetic and venous ulcers versus acute wounds. J Invest Dermatol 111: 850-857, 1998.

22. Szpaderska AM, Egozi EI, Gamelli RL and DiPietro LA: The effect of thrombocytopenia on dermal wound healing. J Invest Dermatol 120: 1130-1137, 2003.

23. Schäfer M and Werner S: Oxidative stress in normal and impaired wound repair. Pharmacol Res 58: 165-171, 2008.

24. Martin P: Wound healing - aiming for perfect skin regeneration. Science 276: 75-81, 1997.

25. Martin P and Leibovich SJ: Inflammatory cells during wound repair: The good, the bad and the ugly. Trends Cell Biol 15: 599-607, 2005.

26. Weiss SJ: Tissue destruction by neutrophils. N Engl J Med 320: 365-376, 1989.

27. Campisi J: Replicative senescence: An old lives' tale? Cell 84: 497-500, 1996

28. Mendez MV, Stanley A, Park HY, Shon K, Phillips T and Menzoian JO: Fibroblasts cultured from venous ulcers display cellular characteristics of senescence. J Vasc Surg 28: 876-883, 1998.

29. Wenk J, Foitzik A, Achterberg V, Sabiwalsky A, Dissemond J, Meewes C, Reitz A, Brenneisen P, Wlaschek M, Meyer-Ingold W and Scharffetter-Kochanek K: Selective pick-up of increased iron by deferoxamine-coupled cellulose abrogates the iron-driven induction of matrix-degrading metalloproteinase 1 and lipid peroxidation in human dermal fibroblasts in vitro: A new dressing concept. J Invest Dermatol 116: 833-839, 2001.

30. Eming SA, Smola $\mathrm{H}$ and Krieg T: Treatment of chronic wounds: State of the art and future concepts. Cells Tissues Organs 172: 105-117, 2002.

31. Martin GS: Sepsis, severe sepsis and septic shock: Changes in incidence, pathogens and outcomes. Expert Rev Anti Infect Ther 10: 701-706, 2012.

32. Martin GS, Mannino DM, Eaton S and Moss M: The epidemiology of sepsis in the United States from 1979 through 2000. N Engl J Med 348: 1546-1554, 2003. 